\title{
The role of tumor vascularisation in benign and malignant cardiovascular neoplasms: A comparison of cardiac myxoma and sarcomas of the pulmonary artery
}

\author{
ANDREAS GAUMANN $^{1}$, GERSON STRUBEL ${ }^{1}$, BEATA BODE-LESNIEWSKA ${ }^{2}$, \\ IRENE SCHMIDTMANN $^{3}$, JÖRG KRIEGSMANN ${ }^{5}$ and C. JAMES KIRKPATRICK ${ }^{4}$
}

\begin{abstract}
${ }^{1}$ Institute of Pathology, University Clinic Regensburg, Franz-Josef Strauss Allee 11, D-93053 Regensburg, Germany;
${ }^{2}$ Institute of Surgical Pathology University Zürich, Schmelzbergstr. 12, CH-8091 Zürich, Switzerland; Institutes of

${ }^{3}$ Biometry, Epidemiology and Informatics and ${ }^{4}$ Pathology, University Clinic Mainz, Langenbeckstr. 1, D-55131 Mainz,

${ }^{5}$ Center for Histology, Zytology and Molecular Diagnostics, Max-Planck Str. 18+20, D-54296 Trier, Germany
\end{abstract}

Received December 12, 2007; Accepted February 4, 2008

DOI: 10.3892/or_00000008

\begin{abstract}
Neoangiogenesis is involved in the development and progression of malignant tumors. Vascular endothelial growth factor (VEGF) and its receptors have been designated a central part in this process. Since the significance of the assessment of angiogenesis in soft tissue tumors is still a matter of debate, we investigated the vascularisation of cardiac myxomas and compared it with pulmonary artery sarcomas (PAS). Angiogenesis in 18 PAS and 20 myxomas was assessed by morphometry. An immunohistochemical analysis of growth factors and their receptors, HIF-1 $\alpha$ and tumor-associated macrophages (TAM) was performed. Results showed that microvessel density (MVD) in PAS was significantly higher at the border of necrosis versus the areas without necrosis but no difference was observed between PAS and myxomas. Vascular surface area (VSA) and intervascular distances showed a higher vascularisation at the border of necrosis compared to myxomas, which was not significant. VEGF expression was higher in PAS compared to myxomas and was prominent at the sites of necrosis. HIF-1 $\alpha$ expression was marked at the border of necrosis in PAS but was absent in myxomas. Infiltration of the macrophages was significantly higher in myxomas compared to the sarcomas. VEGFR-2 expression was detected in a subset of tumor cells and in blood vessels mainly at the tumor periphery, whereas VEGFR-1 was weakly expressed in the tumors but prominent in the macrophages in cardiac myxomas. PDGF receptors and
\end{abstract}

Correspondence to: Dr Andreas Gaumann, Institut für Pathologie, Klinikum der Universität Regensburg, Franz-Josef Strauss Allee 11, D-93053 Regensburg, Germany

E-mail: andreas.gaumann@klinik.uni-regensburg.de

Key words: angiogenesis, myxoma, sarcoma, morphometry, vascular endothelial growth factor their ligands are strongly present in myxomas and to a lesser extent in the sarcomas. In conclusion, benign and malignant cardiovascular tumors with a different pathophysiology develop a comparable vascularisation. Hypoxia appears to be the strongest inducer of neoangiogenesis in the sarcomas. The expression of receptor tyrosine kinases of the VEGF family provides a basis for an adjuvant therapy.

\section{Introduction}

Cardiac myxomas are the most common primary tumors of the heart. This neoplasm occurs in the 6th decade of life with a slight female predominance. The standard therapy is surgical excision, with a $<3 \%$ recurrence and no metastasis (1). Although myxomas are considered to be benign, malignant myxomas have occasionally been reported in the literature. Emboli in the extremities, viscera and bone occur, but are not considered metastatic histologically. In addition, they are able to regress and normally do not cause death in patients. The clinical signs and symptoms in these patients are nonspecific, such as fever, weight loss and anemia (2). The tumors are histologically characterized by polygonal or spindle-shaped tumor cells embedded in a gelatinous matrix. The neoplasms have a variable cell density with very low mitotic activity. Inflammatory cells, such as macrophages and lymphocytes, hemorrhage and intravascular thrombi are frequently present (3). In contrast, sarcomas of the pulmonary artery (PAS) are rare malignant cardiovascular tumors, which occur mainly in women in the 5 th decade of life (4). These patients have the same non-specific symptoms as the myxoma patients. The patients undergo extensive surgical therapy including pulmonary artery trunk resection and reconstruction as well as adjuvant chemotherapy and/or radiation. Despite extensive surgical and adjuvant therapy most patients show a rapid tumor progression with a fatal outcome within 12 months. Histologically, these tumors are typically spindle-cell neoplasms with a myofibroblastic phenotype and a myxoid background. However, other lines of differentiation have also been described (4). 
Neoangiogenesis, the formation of new capillaries from pre-existing blood vessels, is involved in physiological and pathological processes (5). Vascular endothelial growth factor (VEGF) and its receptors VEGFR-2/-1 are central regulators of angiogenesis and are up-regulated in a variety of tumors (6). VEGFR-2 serves multiple functions, such as endothelial cell proliferation, migration, differentiation and survival, whereas VEGFR-1 is mainly present in monocytes, inducing migration and tissue factor production. The two receptors interact with VEGF-A and VEGFR-1 with VEGF-B and PLGF. In contrast, VEGF-C/D are ligands for VEGFR-3 promoting angiogenesis of lymphatic endothelium (7). Other members of the receptor tyrosine kinase family such as PDGF receptor $\alpha / \beta$ are also involved in tumor vascularisation. The receptors are expressed on pericytes and are stimulated by either PDGF-AA or -BB. They are considered to be involved in vessel maturation and stability and may serve as a target for tumor therapy (8). Numerous studies have provided evidence that microvessel density (MVD) in the area of the most intense neovascularisation is an independent prognostic factor in predicting survival in a variety of tumors, mainly carcinomas. However, the assessment of MVD in spindle-cell tumors still shows conflicting results. This was emphasized in previous consensus conference on the angiogenesis quantification in solid human tumors (9). The assessment of angiogenesis in spindle-cell neoplasms needs further clarification. Therefore, we investigated the vascularisation of two pathophysiologically distinct cardiovascular spindle-cell neoplasms to test whether benign myxomas are less vascularised than aggressive and metastasizing PAS. In addition, we evaluated the expression of receptor tyrosine kinases (RTK), which are involved in the process of angiogenesis.

\section{Materials and methods}

Inclusion criteria. Twenty 'cardiac myxoma' (15 female/5 male) of the atrium were retrieved from the authors' files. Histologically, they are composed of typical sheet-like myxoma cells with abundant eosinophilic cytoplasm and inconspicuous nuclei/nucleoli embedded in a strong myxoid background. Clinically, all myxoma were sporadic and not related to the Carney syndrome complex. Ten myxoma were located in the left and 5 in the right atrium and the remaining 5 cases were unspecified. They measured from 2.1 to $8.2 \mathrm{~cm}$. The age ranged from 24 to 74 years (mean 57). Patient followup time ranged from 32 to 195 months with a mean follow-up time of 121 months. One patient developed local recurrence with a poor survival after 12 months. Two additional patients had a poor survival outcome during the follow-up time but this outcome was unrelated to the disease. Seven patients survived without disease during the follow-up period. Ten patients were lost through follow-up. No adjuvant therapy was applied. Histologically, 19/20 cases showed the typical appearance of polygonal cells with scant eosinophilic cytoplasm and indistinct cell borders. None of our cases showed calcification or metaplastic bone formation. No mitotic activity, tumor necrosis or nuclear atypia was detected. In contrast, one case demonstrated dense cellularity throughout the specimen with moderate nuclear atypia and few mitoses. This case had a recurrence after 12 months showing a focally marked atypia and an increased mitotic figure count and was thus reclassified as low-grade myxoid sarcoma not otherwise specified (NOS).

Eighteen cases (11 males/7 females) coded as 'pulmonary artery sarcoma' were also taken from the authors' files. The tumors were mesenchymal and were found within the pulmonary artery. The tumors showed nuclear pleomorphism, necrosis and increased mitoses including the presence of atypical mitoses. Metastatic diseases from the other organs or primary sites were ruled out by clinical and radiological examination. Patient ages ranged from 30 to 71 , with a median age of 52 years. Tumor size ranged from 1.5 to $19 \mathrm{~cm}$. Patient follow-up time ranged from 1 to 77 months with a median follow-up time of 14 months. Three patients developed local recurrence over 15 months (mean), 11 metastases to the lung and 1 patient each developed metastases to the brain, pleura, kidney, liver and bone marrow, over 16 months (mean), while 2 patients were free of disease 42 months (mean) after primary surgery. One patient was lost to follow-up. All but one case were completely excised. Twelve patients received chemotherapy and/or radiation. Macroscopically, the specimens had a polypoid intraluminal tumor mass in the main artery or pulmonary trunk extending distally along the branches of the pulmonary arteries. In the histological examination the intraluminal growth pattern of the tumors was confirmed by the microscopic spreading of malignant spindle cells along the intima and a myofibroblastic phenotype. The sarcomas were graded with the FNCLCC grading scheme, based on differentiation, mitotic activity and necrosis. One tumor was grade 1, 10 sarcomas were classified as grade 2 and 7 tumors were grade 3 . The morphological spectrum of the intermediate and high-grade tumors ranged from myxofibrosarcoma-like to storiform pleomorphic malignant fibrous histiocytoma-like. The mitotic activity ranged from 1 to $35 / 10 \mathrm{HPF}$. The proliferation index ranged from 5 to $80 \%$ using Ki-67 staining. Excessive tumor necrosis $(>50 \%)$ was present in 4 cases and necrosis was absent in one case. One case demonstrated focal osseous metaplasia, although no true neoplastic bone or cartilage formation was detected.

Patient data and follow-up were obtained from patient records. The study was performed according to the criteria for the use of patient archive material, as laid down by the ethics committee. Immediately after surgery, the tissue samples were fixed in $4 \%$ formaldehyde and embedded in paraffin.

Immunohistochemical staining procedures. The antibodies used in this study are summarized in Table I. Antigen retrieval was performed using either a microwave $(4 \times 4 \mathrm{~min}$ at $800 \mathrm{~W}$ in citrate buffer, $\mathrm{pH} 6.0)$ or pressure cooker $\left(120^{\circ} \mathrm{C}\right.$ for $\left.5 \mathrm{~min}\right)$. Non-specific binding was blocked with either $20 \%$ goat or rabbit serum in PBS. Antibodies against Factor VIII, CD68 and CD163 were applied by using an immunostainer (Ventana Medical Systems, USA). VEGFR-1, VEGFR-2 and VEGF-A, HIF- $1 \alpha$, PDGF-AA, PDGF-BB and PDGFR- $\alpha / \beta$ were incubated in a moisture chamber overnight at $4^{\circ} \mathrm{C}$. Antibody detection was performed by adding biotinylated secondary antibodies, avidin biotin complex (ABC), and 3,3'-diaminobenzidine (DAB) or amino-ethyl-carbazole (AEC). Besides the 
Table I. Antibody types and sources used in this study.

\begin{tabular}{llllll}
\hline Antibody & Antigen & Dilution & Pretreatment (citrate buffer: pH 6.0) & Company & Clone \\
\hline Rabbit IgG & Factor VIII & $1: 600$ & Microwave, $320 \mathrm{~W}$ for $30 \mathrm{~min}$ & Dako & Polyclonal \\
Rabbit IgG & VEGF & $1: 40$ & Pressure cooker, $120^{\circ} \mathrm{C}$ for $30 \mathrm{~min}$ & Zymed Lab & Polyclonal \\
Mouse IgG2b & HIF-1 $\alpha$ & $1: 500$ & Pressure cooker, $120^{\circ} \mathrm{C}$ for 5 min & Novus Biotech & Hia67 \\
Mouse IgG1 & VEGFR-1 & $1: 500$ & Pressure cooker, $120^{\circ} \mathrm{C}$ for 5 min & DCS Hamburg & Polyclonal \\
Rabbit IgG & VEGFR-2 & $1: 100$ & Pressure cooker, $120^{\circ} \mathrm{C}$ for 5 min & Santa Cruz & Clone A-3 \\
Rabbit IgG & VEGFR-3 & $1: 100$ & Pressure cooker, $120^{\circ} \mathrm{C}$ for 5 min & DCS Hamburg & Polyclonal \\
Rabbit IgG & PDGFR- $\alpha$ & $1: 50$ & Pressure cooker, $120^{\circ} \mathrm{C}$ for 5 min & Cell Signaling & Monoclonal \\
Rabbit IgG & PDGFR- 3 & $1: 100$ & Pressure cooker, $120^{\circ} \mathrm{C}$ for 5 min & Cell Signaling & Monoclonal \\
Rabbit IgG & PDGF-AA & $1: 250$ & Pressure cooker, $120^{\circ} \mathrm{C}$ for 5 min & Santa Cruz & Polyclonal \\
Rabbit IgG & PDGF-BB & $1: 100$ & Pressure cooker, $120^{\circ} \mathrm{C}$ for 5 min & Santa Cruz & Polyclonal \\
Mouse IgG & CD68 & $1: 100$ & Microwave, $320 \mathrm{~W}$ for 30 min & Dako Cytomation & PG-M1 \\
Mouse IgG & CD163 & $1: 100$ & Microwave, $320 \mathrm{~W}$ for 30 min & Novocastra & $10 D 6$ \\
\hline
\end{tabular}

ABC method, factor VIII and VEGF-A stains were performed using the Dako Envision ${ }^{\circledR}$ kit (Dako), coupled with peroxidase and developed with the AEC substrate. In the case of HIF-1 $\alpha$ a tyramide-based amplification kit (CSA-kit ${ }^{\circledR}$, Dako) was applied according to the manufacturer's protocol. The sections were counterstained with hematoxylin and mounted with Aquatex $^{\circledR}$ (Merck, Germany). For the negative controls, primary antibodies were omitted or replaced by non-specific immunoglobulins.

Alkaline phosphatase immunohistochemical staining. Double-labeling was performed using the Dako Envision kit with AEC for factor VIII and then the ABC-alkaline phosphatase method with the antibody against VEGFR-2. After washing with Tris the slides were incubated for $1 \mathrm{~h}$ at room temperature (RT) with the primary antibody (VEGFR-2) in Tris ( $\mathrm{pH}$ 7.6). The sections were then rinsed in Tris ( $\mathrm{pH} 7.6)$ and incubated for $30 \mathrm{~min}$ at RT with a secondary goat antimouse antibody (Jackson Immunoresearch, USA) in a 1:200 dilution in Tris ( $\mathrm{pH}$ 7.6). The avidin-biotin complex (Vector Laboratories, USA) was then incubated for $30 \mathrm{~min}$ at RT in a 1:100 Tris ( $\mathrm{pH} 7.6$ ) followed by the addition of the NBT/ BCIP chromogen with Tris ( $\mathrm{pH}$ 9.7). Color development was stopped by adding Tris ( $\mathrm{pH} 7.6)$.

Microscopic and statistical analysis. The specimens were analyzed by light microscopy (Zeiss AG) by two different and independent observers (A.G. and G.S.). The extent of the immunostaining was estimated by counting the number of positive cells/10 high-power fields (HPF). Results were classified as: 0 , negative; $1,1-25 \%$ of cells positive; $2,26-50 \%$ of cells positive; $3,51-75 \%$ of cells positive; $4,76-100 \%$ of cells positive. Concerning factor VIII staining two independent observers (A.G. and G.S.) examined up to 5 different sections per myxoma/sarcoma and identified up to 5 areas of the highest vascularisation in every section. These hot-spot areas were digitized with an image analysis program (Axiovision, Zeiss AG). All examinations were performed without any knowledge of clinical parameters or prognostic factors.
Within the hot-spots the vascular densities were evaluated by counting the microvessels based on the criteria of Weidner. In addition, the vascular surface areas were analyzed by using printouts of the images. A transparent grid was put on top and the squares crossing the vessel were counted, related to the total amount of squares and depicted in \%. The intervascular distances were measured by an image analysis program (KS 300, Zeiss AG). The smallest distances between neighboring vessels were measured (mm). Distribution of the microvessel count and vascular surface area are displayed in the box plots. The distribution of intervascular distances is depicted in a cumulative frequency polygon. These distributions include all available values from the patients.

Statistical analyses and graphic displays were performed using SigmaPlot (10.0, SPSS, Germany) and the PROC MIXED procedure of SAS software (9.1.3). In order to assess the differences between myxomas and sarcomas we fitted general mixed linear models. These models allow for the analysis of dependent observations including multiple specimens from each patient. We quoted the mean and standard error of mean (SEM). The SAS PROC MIXED analysis uses a restricted maximum likelihood estimation, which leads to different estimates for group means and SEM depending on the total sample. The objective analysis was exploratory. Therefore, we did not adjust for multiple testing. We termed differences statistically significant at $\mathrm{p}<0.05$.

\section{Results}

Evaluation of the microvessel count. Only sections with an accurate factor VIII staining were evaluated (Fig. 2A and B). Three myxomas and one pulmonary artery sarcoma did not meet the criteria of accurate staining and were excluded from the morphometry analysis. Two myxomas/sarcomas showed extensive hemorrhage/necrosis so that only one area of the highest vascularisation was identified. The microvessel count in the PAS group was analyzed in areas without necrosis and at the border of necrosis. When comparing the areas without necrosis $(26.42 \pm 4.55)$ and areas at the border of necrosis 
A
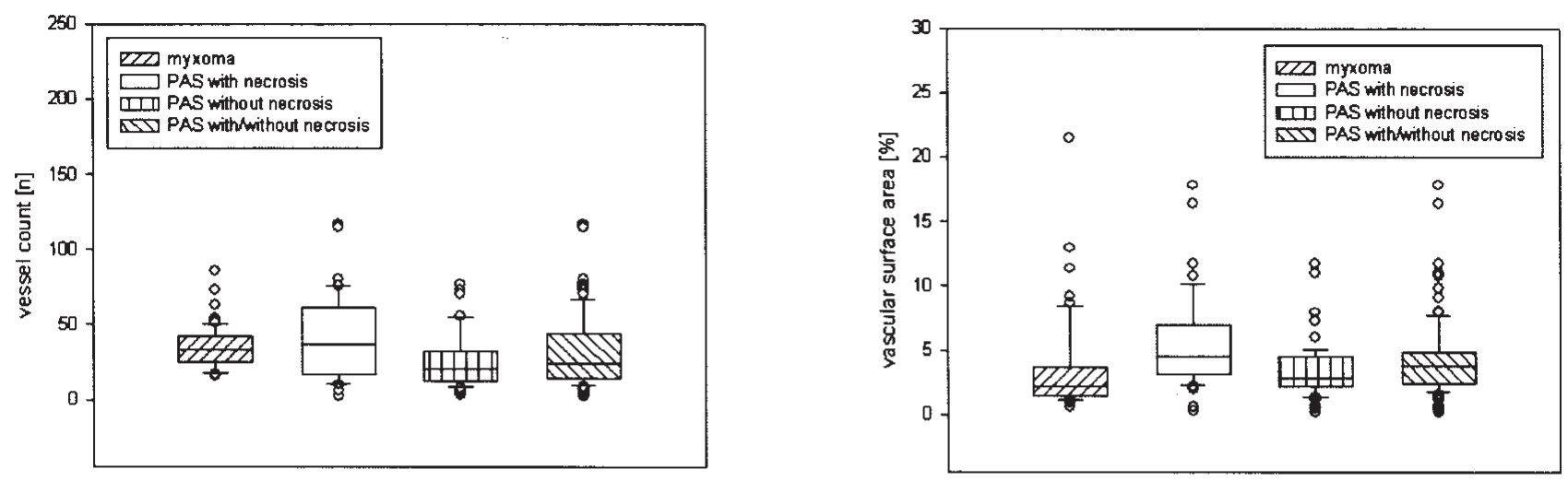

C
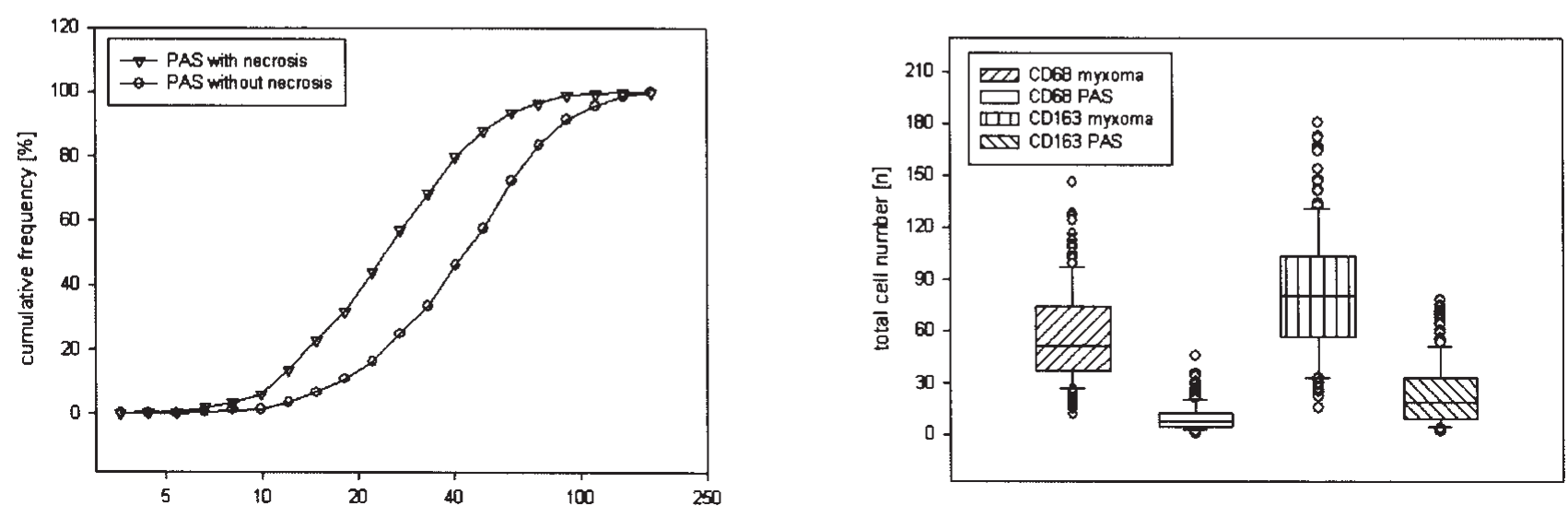

Figure 1. Comparison of the microvessel count showed a higher vessel density for sarcomas at the sites of necrosis when compared to those without necrosis and myxomas (A). Comparable results were obtained for the vascular surface area for sarcomas and myxomas. A statistically significant difference was observed between the sarcomas with necrosis and those without necrosis (B). Intervascular distances differed significantly between sarcomas with and without necrosis $(\mathrm{C})$. The infiltration of tumor-associated macrophages was significantly higher in myxomas compared to the sarcomas (D).

(38.94 \pm 4.62$)$ a statistically significant difference was observed ( $\mathrm{p}=0.0011)$ (Table II and Fig. 1A). PAS indicated a statistically significant higher vessel count in the areas with necrosis (38.59 44.48$)$ compared to the myxomas $(24.86 \pm 4.39)$ ( $\mathrm{p}=0.037)$. However, the comparison of the myxoma group $(24.79 \pm 2.70)$ with PAS without necrosis $(26.5 \pm 2.7, \mathrm{p}=0.66)$ showed no significant difference $(\mathrm{p}=0.45)$. When comparing all the PAS (32.42 \pm 3.44$)$ with the cardiac myxoma group no significant difference $(\mathrm{p}=0.16)$ was observed.

Evaluation of the vascular surface area. The morphometry of the vascular surface area (VSA) displayed $4.30 \pm 0.58 \%$ of the total tumor area for PAS with and without necrosis and a lower VSA of $3.70 \pm 0.68 \%$ for the myxoma group, which was not significantly different $(\mathrm{p}=0.51$ ) (Table II). When the VSA at the sites of necrosis of PAS $(4.98 \pm 0.76 \%)$ were compared to the VSA of cardiac myxomas $(3.71 \pm 0.75 \%)$ no statistically significant differences $(\mathrm{p}=0.242)$ were seen. PAS without necrosis showed levels $(3.63 \pm 0.69 \%)$ comparable to the myxoma group $(3.71 \pm 0.67 \%)$, which was not statistically different $(\mathrm{p}=0.94)$. However, a comparison of VSA in PAS at the sites of necrosis $(5.23 \pm 0.51 \%)$ and without necrosis $(3.46 \pm 0.49 \%)$ indicated a significant difference $(\mathrm{p}=0.0021)$ (Fig. 1B).

Evaluation of the intervascular distances. The intervascular distances showed no significant difference between the PAS groups with and without necrosis and the cardiac myxomas [PAS $(75.99 \pm 6.52)$ vs. myxomas $(82.26 \pm 7.66 \mu \mathrm{m}),(\mathrm{p}=0.537)$ (Table II)]. When comparing PAS at the sites of necrosis slightly lower intervascular distances $(72.44 \pm 6.55 \mu \mathrm{m})$ were detected compared to the cardiac myxomas $(82.26 \pm 7.10 \mu \mathrm{m})$, which was not statistically significant $(\mathrm{p}=0.317)$. When comparing PAS without necrosis $(89.73 \pm 7.47 \mu \mathrm{m})$ with the cardiac myxomas $(82.26 \pm 6.59 \mu \mathrm{m})$, no difference was detected $(\mathrm{p}=0.46)$. The comparison of PAS at the sites of necrosis $(69.25 \pm 8.39 \mu \mathrm{m})$ and without necrosis $(87.36 \pm 9.29 \mu \mathrm{m})$, however, showed a significant difference for this parameter $(\mathrm{p}=0.0203)$ (Fig. 1C) (Table III).

Evaluation of tissue-associated macrophages (TAM). The total cell count of tumor-associated macrophages was 
Table II. Microvascular morphometry in cardiac myxomas.

\begin{tabular}{|c|c|c|c|c|}
\hline Patient no. & Location size $(\mathrm{cm})$ & Microvessel count & Vascular surface area & Intervascular distances \\
\hline 1 & $\begin{array}{l}\text { Right atrium } \\
5.3 \times 4.2 \times 3.4\end{array}$ & $16.5 \pm 2.2$ & $1.52 \pm 0.20$ & $103.66 \pm 5.92$ \\
\hline 2 & $\begin{array}{l}\text { Left atrium } \\
4 \times 3 \times 3\end{array}$ & $28.67 \pm 5.17$ & $3.39 \pm 2.04$ & $61.82 \pm 5.27$ \\
\hline 3 & $\begin{array}{l}\text { Right atrium } \\
8.2 \times 6 \times 4.5\end{array}$ & $23.33 \pm 1.76$ & $4.24 \pm 0.33$ & $85.45 \pm 5.71$ \\
\hline 4 & $\begin{array}{l}\text { Left atrium } \\
4.8 \times 3.5 \times 2.4\end{array}$ & $29.0 \pm 2.08$ & $2.08 \pm 0.71$ & $78.99 \pm 4.86$ \\
\hline 5 & $\begin{array}{l}\text { Left atrium } \\
5\end{array}$ & $26.00 \pm 1.15$ & $14.54 \pm 3.65$ & $69.38 \pm 6.03$ \\
\hline 6 & $\begin{array}{l}\text { Right atrium } \\
6 \times 5 \times 3.5\end{array}$ & $25.67 \pm 2.73$ & $1.84 \pm 0.29$ & $90.57 \pm 5.55$ \\
\hline 7 & $\begin{array}{l}\text { Left atrium } \\
4.5 \times 3.5 \times 1.8\end{array}$ & $19 \pm 2.89$ & $6.05 \pm 2.69$ & $88.79 \pm 6.35$ \\
\hline 8 & $\begin{array}{l}\text { Left atrium } \\
4\end{array}$ & 22 & 4.98 & $88.06 \pm 7.54$ \\
\hline 9 & $\begin{array}{l}\text { Left atrium } \\
8.2 \times 6 \times 2.5\end{array}$ & Excluded & Excluded & Excluded \\
\hline 10 & $\begin{array}{l}\text { Left atrium } \\
2.1 \times 2 \times 2\end{array}$ & 40 & 2.16 & $60.27 \pm 5.84$ \\
\hline 11 & $\begin{array}{l}\text { Right atrium } \\
2.5 \times 1.5 \times 0.4\end{array}$ & $32.67 \pm 0.88$ & $2.95 \pm 0.99$ & $66.56 \pm 3.91$ \\
\hline 12 & $\begin{array}{l}\text { Left atrium } \\
5 \times 4 \times 2.5\end{array}$ & $27.6 \pm 3.56$ & $2.24 \pm 0.54$ & $81.80 \pm 3.49$ \\
\hline 13 & $\begin{array}{l}\text { Atrium } \\
7.5 \times 5.2 \times 2.7\end{array}$ & $14.5 \pm 0.5$ & $6.05 \pm 2.65$ & $100.83 \pm 10.7$ \\
\hline 14 & $\begin{array}{l}\text { Left atrium } \\
3.3 \times 2.1 \times 2\end{array}$ & Excluded & Excluded & Excluded \\
\hline 15 & $\begin{array}{l}\text { Atrium } \\
\max .5 .5\end{array}$ & $26.5 \pm 5.37$ & $2.11 \pm 0.48$ & $81.84 \pm 3.49$ \\
\hline 16 & $\begin{array}{l}\text { Right atrium } \\
7 \times 5 \times 3\end{array}$ & $23.5 \pm 5.5$ & $1.59 \pm 0.67$ & $79.04 \pm 5.85$ \\
\hline 17 & $\begin{array}{l}\text { Left atrium } \\
2.7 \times 2.5 \times 1.5\end{array}$ & 20 & 1.46 & $132.84 \pm 17.43$ \\
\hline 18 & $\begin{array}{l}\text { Atrium } \\
3.6 \times 2.8 \times 1.4\end{array}$ & $24.33 \pm 3.18$ & $2.03 \pm 0.454$ & $87.72 \pm 4.41$ \\
\hline 19 & $\begin{array}{l}\text { Left atrium } \\
2.5 \times 1 \times 0.9\end{array}$ & $27.33 \pm 5.36$ & $1.43 \pm 0.13$ & $94.88 \pm 6.48$ \\
\hline 20 & $\begin{array}{l}\text { Atrium } \\
7.2 \times 6.2 \times 4.5\end{array}$ & Excluded & Excluded & Excluded \\
\hline
\end{tabular}


Table III. Microvascular morphometry in pulmonary artery sarcoma.

\begin{tabular}{|c|c|c|c|c|c|c|}
\hline \multirow[b]{2}{*}{ Patient no. } & \multicolumn{2}{|c|}{ Microvessel count } & \multicolumn{2}{|c|}{ Vascular surface area } & \multicolumn{2}{|c|}{ Intervascular distances } \\
\hline & No necrosis & With necrosis & No necrosis & With necrosis & No necrosis & With necrosis \\
\hline 1 & $35.71 \pm 6.45$ & $16.10 \pm 3.04$ & $6.77 \pm 1.97$ & $2.72 \pm 0.48$ & $56.26 \pm 2.71$ & $127.87 \pm 6.75$ \\
\hline 2 & $30.00 \pm 4.00$ & $9.00 \pm 1.73$ & $4.51 \pm 0.61$ & $2.31 \pm 1.24$ & $79.24 \pm 6.75$ & $171.40 \pm 25.42$ \\
\hline 3 & Excluded & Excluded & Excluded & & & \\
\hline 4 & $29.00 \pm 15.00$ & $12.00 \pm 1.97$ & $3.09 \pm 0.65$ & $1.71 \pm 0.54$ & $129.27 \pm 12.50$ & $118.77 \pm 9.72$ \\
\hline 5 & $11.50 \pm 0.50$ & $11.33 \pm 2.03$ & $2.93 \pm 0.37$ & $5.32 \pm 1.38$ & $165.91 \pm 19.94$ & $178.04 \pm 25.69$ \\
\hline 6 & $30.00 \pm 5.69$ & $54.00 \pm 19.00$ & $3.52 \pm 0.99$ & $3.65 \pm 0.77$ & $74.03 \pm 5.65$ & $49.60 \pm 5.74$ \\
\hline 7 & $77.50 \pm 250$ & $33.50 \pm 8.99$ & $4.72 \pm 0.10$ & $2.70 \pm 0.35$ & $47.88 \pm 2.79$ & $81.57 \pm 3.68$ \\
\hline 8 & & $60.00 \pm 6.40$ & & $4.83 \pm 1.43$ & & $67.50 \pm 2.83$ \\
\hline 9 & & $15.75 \pm 1.38$ & & $7.32 \pm 1.31$ & & $71.74 \pm 7.51$ \\
\hline 10 & $17.00 \pm 5.13$ & 20 & $7.07 \pm 2.57$ & 2.1 & $55.12 \pm 4.50$ & $40.11 \pm 9.73$ \\
\hline 11 & $58.20 \pm 3.38$ & $4.34 \pm 0.17$ & $37.3 \pm 1.72$ & & & \\
\hline 12 & & $26.50 \pm 6.64$ & & $2.48 \pm 0.18$ & & $74.31 \pm 5.44$ \\
\hline 13 & & $25.75 \pm 3.07$ & & $4.30 \pm 0.41$ & & $54.71 \pm 2.64$ \\
\hline 14 & $52.33 \pm 6.69$ & $34.67 \pm 3.18$ & $5.47 \pm 0.93$ & $3.97 \pm 0.2$ & $36.39 \pm 1.55$ & $50.61 \pm 4.95$ \\
\hline 15 & $80.33 \pm 12.01$ & & $8.75 \pm 0.57$ & & $24.56 \pm 1.11$ & \\
\hline 16 & $30.00 \pm 3.61$ & $20.00 \pm 3.00$ & $3.03 \pm 0.49$ & $3.6 \pm 1.5$ & $42.01 \pm 2.97$ & $52.70 \pm 4.20$ \\
\hline 17 & $14.50 \pm 0.5$ & $31.00 \pm 7.63$ & $11.00 \pm 5.40$ & $3.73 \pm 0.65$ & $52.63 \pm 5.79$ & $44.02 \pm 2.55$ \\
\hline 18 & 12 & $23.25 \pm 5.51$ & 3.1 & $2.68 \pm 0.29$ & $85.30 \pm 13.20$ & $59.43 \pm 3.22$ \\
\hline
\end{tabular}

evaluated in PAS and cardiac myxoma by two different macrophage markers. Using the two markers we observed a significantly higher infiltration of TAM $(\mathrm{p}<0.0001)$ in cardiac myxomas (Fig. 2C) when compared to sarcomas of the pulmonary artery (Fig. 2D). For CD68 58.54 \pm 5.62 macrophages were found in cardiac myxomas and only $11.22 \pm 6.62$ TAM were detected in PAS. The other marker, CD163 indicated $87.92 \pm 6.92$ TAM in the myxoma group but only $24.81 \pm 7.83$ macrophages in the PAS group (Fig. 1D).

Evaluation of HIF-1 a expression in myxomas and sarcomas. The HIF-1 $\alpha$ protein, which is stabilized under hypoxic conditions and transferred to the nucleus was immunohistochemically detected. The molecule was prominent at the border of necrosis in the nucleus of the sarcoma cells (Fig. 2F) and to a lesser extent in the cytoplasm, whereas in the areas without necrosis it was only expressed in a scattered fashion. However, HIF-1 $\alpha$ was not detectable in the cardiac myxoma cells (Fig. 2E).

Evaluation of the growth factor and growth factor receptor expression. The analysis of VEGF expression showed a weak ( $15 \%, 3$ cases) to moderate $(30 \%, 6$ cases) staining in the tumor cells of the cardiac myxomas (Fig. 3A), while 11 cases $(55 \%)$ were completely negative for VEGF. In addition, numerous mononuclear infiltrating cells were stained with antibodies against VEGF as well as a few endothelial cells. In contrast, PAS showed a marked staining for VEGF in 14 cases $(77.78 \%)$ and a moderate expression in 3 cases $(16.67 \%)$ (Fig. 3B). Few macrophages and endothelial cells expressed VEGF.
A marked expression of VEGFR-1 was observed in 6 myxoma cases $(30 \%)$. A moderate expression of VEGFR-1 was detected in 5 cases $(25 \%)$ and the signal was weak in 2 $(10 \%)$ and absent in 1 case (5\%) (Fig. 3C). VEGFR-1 was also present in infiltrating mononuclear cells. In contrast, the receptor was only weakly expressed in endothelial cells. PAS showed a moderate expression in 4 cases $(22.22 \%)$, weak staining in 8 cases $(44.44 \%)$ and no expression in 6 cases $(33.33 \%)$. Mononuclear cells were also stained with VEGFR-1, but to a much lesser extent than the myxomas (Fig. 3D). VEGFR-1 was also present in tumor-associated vessels in 15 cases.

Cardiac myxomas displayed a marked expression of VEGFR-2 in 4 cases (20\%) and a very marked expression in 5 cases $(25 \%)$ in the tumor cells (Fig. 3E). A moderate expression was present in 6 cases $(30 \%)$, a weak expression was observed in 4 cases $(20 \%)$ while 1 case $(5 \%)$ showed no signal for VEGFR-2. Most cases showed a strong expression of VEGFR-2 in tumor-associated vessels. VEGFR-2 was not detected in 7 cases $(38.89 \%)$ and was only weakly present in 5 cases $(27.78 \%)$ of PAS. Two cases $(11.11 \%)$ showed a marked and 4 cases $(22.22 \%)$ a very marked expression of VEGFR-2 in the tumor cells (Fig. 3F). Double labeling experiments showed that VEGFR-2 positive endothelial cells were evident in a subset of microvessels mostly at the tumor border and to a lesser extent in the tumor center (Fig. 4A and B). VEGFR-3 was only present in a few endothelial cells, was weakly expressed in the spindle-shaped tumor cell component of 8 myxoma cases $(40 \%)$ and was moderate in 1 case $(5 \%)$. The remaining 11 cases $(55 \%)$ were negative. PAS did not show any signal for VEGFR-3 in all 18 cases. 

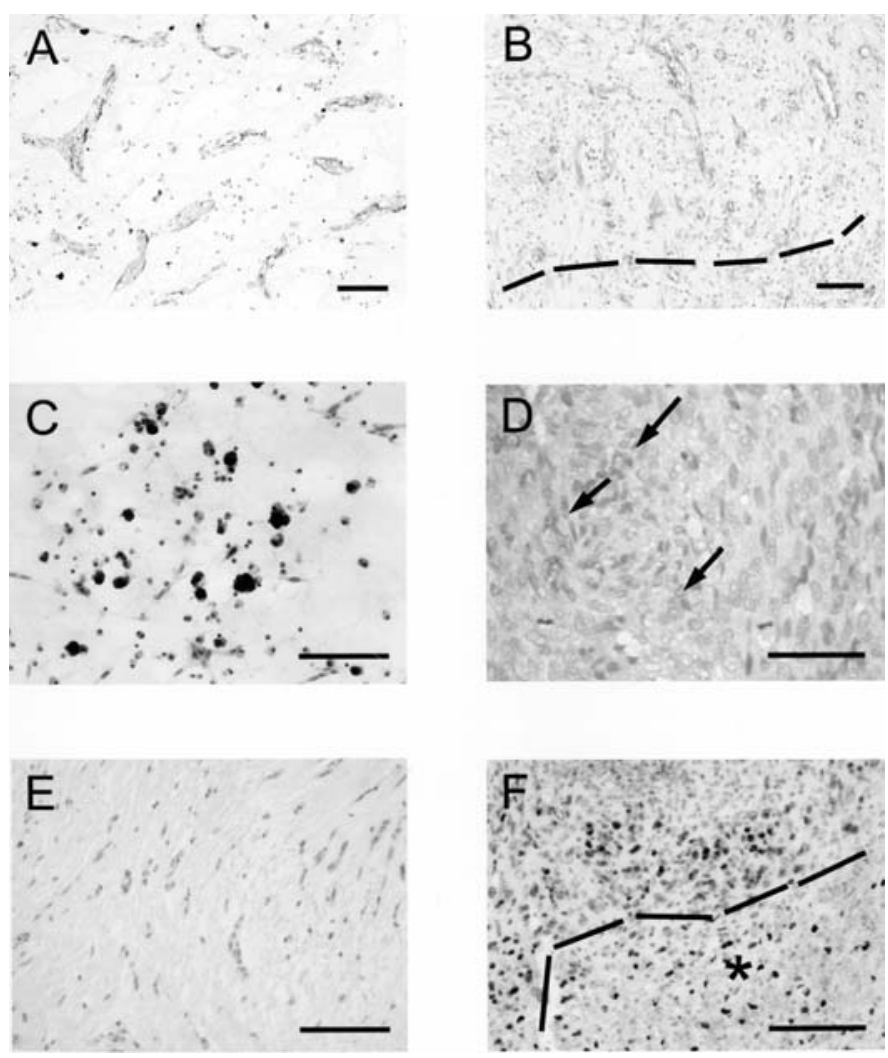

Figure 2. Factor VIII staining shows a prominent vasculature in cardiac myxomas with a typical perivascular arrangement of myxoma cells and an abundant myxoid background (A) (ABC, AEC, magnification 10X, Bar $100 \mu \mathrm{m}$ ). Abundant hemorrhage and necrosis in a case of pulmonary artery sarcoma with myofibroblastic differentiation. Note the prominent vascularisation at the border of the necrosis (B) (ABC, AEC, magnification $10 \mathrm{X}$, Bar $100 \mu$ m). Numerous infiltrating macrophages in myxomas (C) (ABC, AEC, magnification 20X, Bar $100 \mu \mathrm{m}$ ) and only a few macrophages in sarcomas (D) ( $\rightarrow$ ) (ABC, AEC, magnification $40 \mathrm{X}$, Bar $50 \mu \mathrm{m})$. F shows the abundant nuclear staining of HIF-1 $\alpha$ at the border of necrosis (*) in a sarcoma case versus its absence in myxomas (E) (ABC, AEC, magnification 20X, Bar $100 \mu \mathrm{m})$.
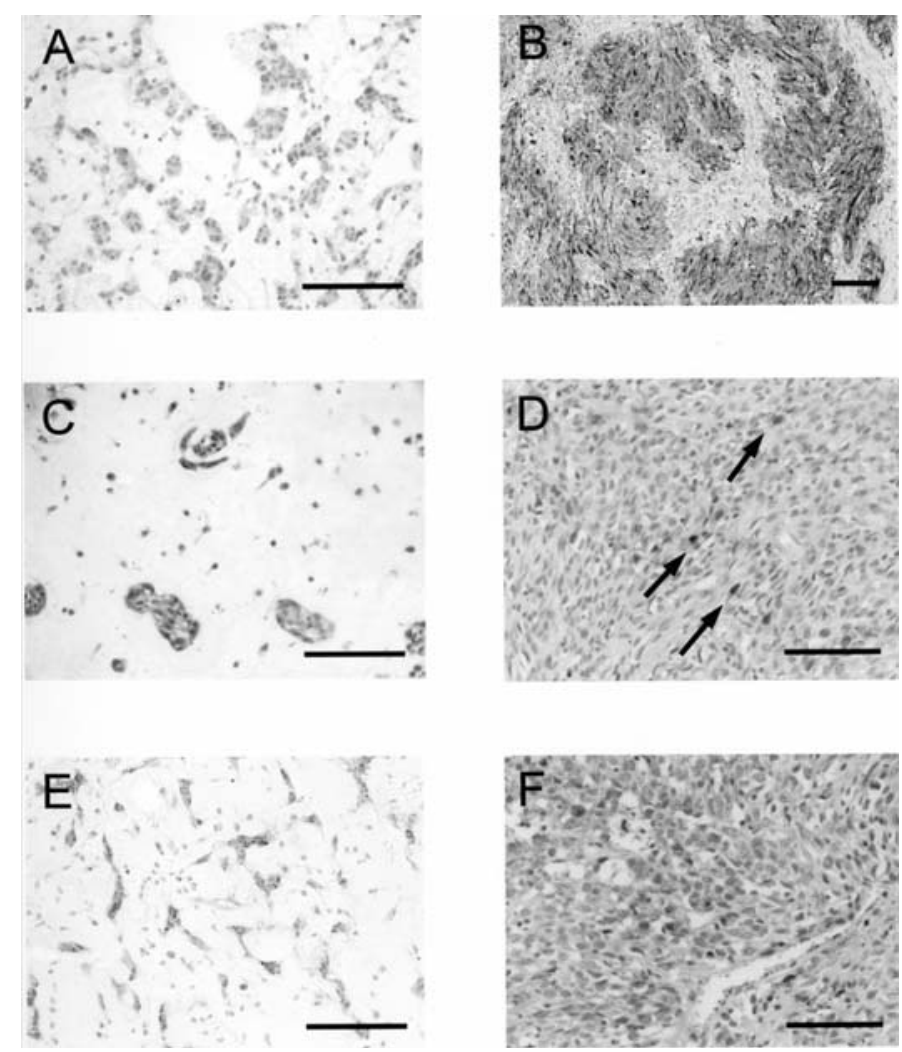

Figure 3. (A) A moderate expression of VEGF in myxoma cells is shown. (B) A very marked expression at the sites of necrosis in sarcoma cells is shown (Envision ${ }^{\circledR}$, AEC, magnification 20X, Bar $100 \mu \mathrm{m}$ ). VEGFR-1 was expressed in myxoma cells (C) but was mostly negative in the sarcoma cells (D). In addition, infiltrating mononuclear cells were positive for VEGFR-1 $\rightarrow$ (ABC, AEC, magnification $20 \mathrm{X}$, Bar $100 \mu \mathrm{m})$. VEGFR-2 was present in myxomas $(\mathrm{E})$ and sarcomas $(\mathrm{F})$ as well as in the tumor vasculature (ABC, AEC, magnification 20X, Bar $100 \mu \mathrm{m}$ ). 

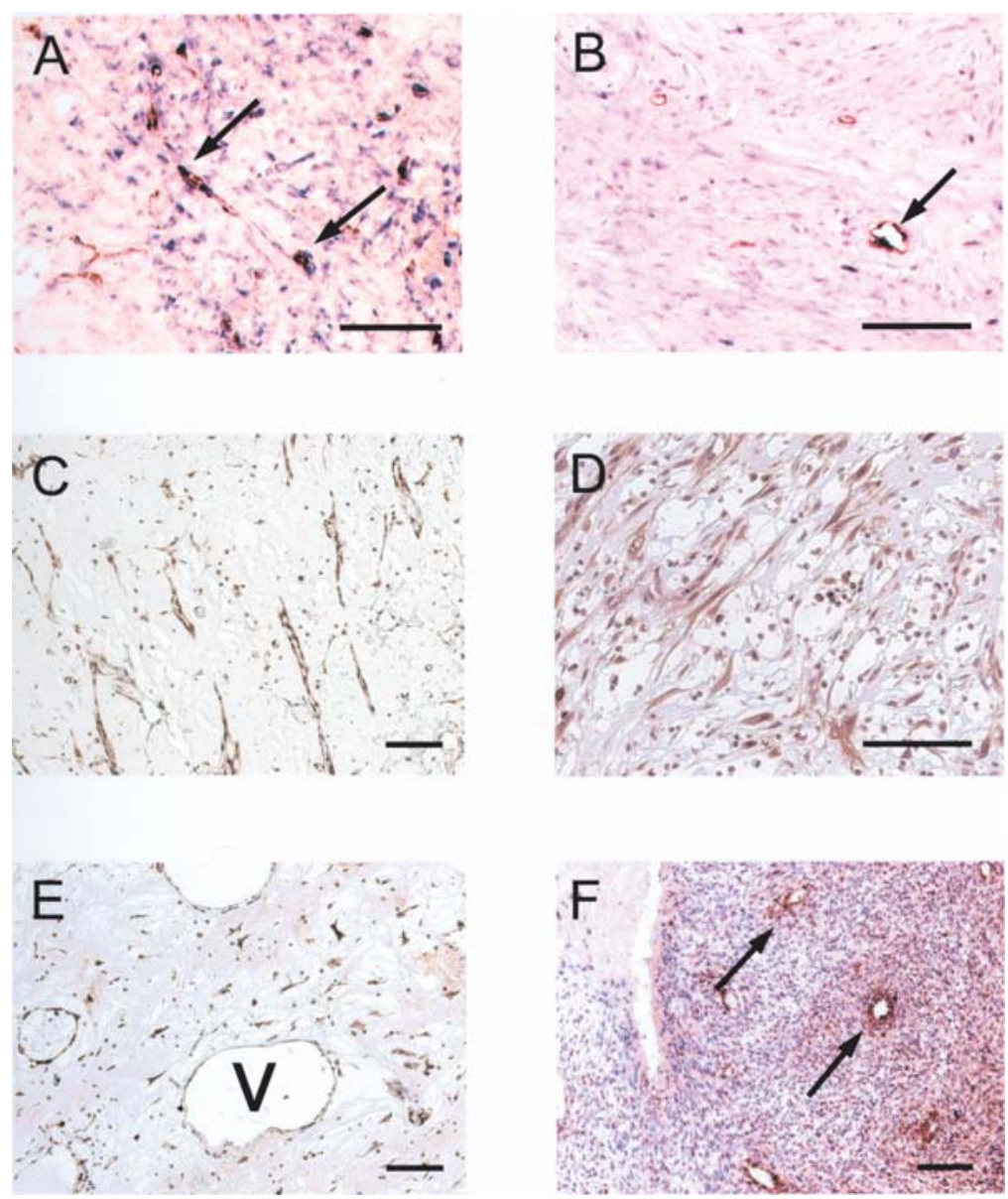

Figure 4. (A and B) A subset of tumor vessels (factor VIII, red), which co-express VEGFR-2 (black) in the myxomas (A) and the sarcomas (B) (Envision ${ }^{\circledR}$, AEC, magnification 20X, Bar $100 \mu \mathrm{m})$ is shown. The marked expression of PDGFR- $\alpha$ in a myxoma case $(\mathrm{C})(\rightarrow)(\mathrm{ABC}, \mathrm{AEC}$, magnification $10 \mathrm{X}$, Bar 100 $\mu \mathrm{m}$ ) and a sarcoma case (D) (ABC, AEC, magnification 20X, Bar $100 \mu \mathrm{m}$ ) is shown. PDGFR- $\beta$ expression in myxomas (E) and sarcomas (F) showing a prominent expression in pericytes $\rightarrow$ (ABC, AEC, magnification 10X, Bar $100 \mu \mathrm{m})$.

PDGFR- $\alpha$ was markedly expressed in 7 cases (35\%) and very markedly in 8 cases (40\%) of myxomas (Fig. 4C). A moderate expression was detected in only 4 cases (20\%). Comparable results were detected for its ligand, PDGF-AA, with 7 cases $(35 \%)$ showing a marked and 6 cases $(30 \%)$ a very marked expression. However, 5 cases (25\%) showed no expression of PDGF-AA. In the sarcoma cells PDGFR- $\alpha$ was markedly expressed in 8 cases $(44,44 \%)$, was moderate in 1 case $(5.56 \%)$ and weak in 6 cases (33.33\%) (Fig. 4D). The ligand PDGF-AA was detected in 9 cases $(50 \%)$ but was absent in 4 cases $(22.22 \%)$.

PDGFR- $\beta$ was markedly present in 13 myxoma cases $(65 \%)$, only moderately expressed in 3 cases $(15 \%)$, while absent in 3 additional cases (15\%) (Fig. 4E). The ligand PDGF-BB was absent in 8 cases (40\%) but showed a marked expression in 2 cases $(10 \%)$ and a very marked expression in 5 cases $(25 \%)$. PDGFR- $\beta$ was markedly expressed in 12 PAS $(66.67 \%)$ but was absent in 3 cases $(16.67 \%)$. A weak/ moderate expression was observed in 3 sarcoma cases (16.67\%). PDGFR- $\beta$ was present in numerous pericytes accompanying tumor-associated vessels (Fig. 4F). The ligand PDGF-BB was very markedly expressed in 10 sarcomas $(55.56 \%)$ and markedly expressed in 2 cases $(11.11 \%)$. A weak to moderate expression was observed in 3 additional cases $(16.67 \%)$.

\section{Discussion}

The formation of new blood vessels in tumors is a complex mechanism driven by a variety of different molecules. VEGF promotes blood vessel formation by interacting with VEGF receptors. Several stimulators have been reported for VEGF including hypoxia (10), cobalt chloride and a variety of growth factors, including interleukins, TGF- 31 and PDGF-BB. Since the anti-angiogenic therapy targeting these molecular pathways has become a promising tool for cancer treatment, the investigation of tumor microvascularisation and its underlying mechanisms is of fundamental importance.

In our study, we were able to confirm the results by Kono et al (11) and Sakamoto et al (12) showing the expression of VEGF in all myxoma cases, although the expression was lower than in the cases of PAS. This may be attributed to a different methodology, since we used an amplification technique for immunohistochemistry leading to a relative underestimation of VEGF expression compared to the sarcoma group. In addition, we observed a strong presence of VEGF in numerous infiltrating mononuclear cells of the myxoma group. The number of these cells was assessed and it was shown that they were significantly higher in the myxoma group compared to the sarcoma group. Thus, the mononuclear infiltrate is the main source of VEGF production in cardiac myxoma. The 
potential role of these tumor-associated macrophages (TAM) and their association with MVD has been described before (13). Furthermore, MCP-1, a potent chemoattractant produced by macrophages, is able to promote tumor angiogenesis (14). IL- 6 has also been described to induce the expression of VEGF (15). MCP-1 and IL-6 are known to be expressed in myxoma of the heart and a correlation with MVD has been shown for MCP-1, (16) emphasizing that the induction of angiogenesis in these tumors is a complex interplay of several different molecules. In contrast, PAS showed only a sparse infiltrate of TAM compared to the myxomas. However, HIF-1 $\alpha$ was strongly present at the border of necrosis in the sarcoma group but completely absent in the myxoma group. Since it is known that this molecule is able to directly induce VEGF transcription by binding to the hypoxia response element of the VEGF promoter, it is obvious that hypoxia is the main driving force of angiogenesis in PAS. Notably, we were able to show that MVD, VSA and IVD tend to be higher at the sites of necrosis in the sarcoma versus the myxoma group. However, a statistically significant difference was not detected between the two groups. Thus, it can be concluded from these findings that angiogenesis is slightly stronger when induced by hypoxia than cytokines released from TAM. It has recently been published that the VEGF transcription in macrophages is activated in a comparable manner as in hypoxia (17).

However, no difference was found between sarcomas and myxomas with respect to the examined microvascular parameters. It is a common belief that benign neoplasms tend to be less vascularised than malignant tumors. In a review, Hlatky et al summarized the data available on MVD. They stated that this method may help in assessing the disease stage, metastasis, recurrence and survival. They also mentioned that differences in methodology may be responsible for no or even negative correlation of MVD and these variables (18). These assumptions are made mainly based on studies of carcinomas. Comparatively little is known about spindle-cell neoplasms and MVD. In a series of uterine leiomyoma and leiomyosarcoma no significant difference of MVD was observed between these two groups (19). Others, however, found a significantly higher vascularisation in uterine leiomyosarcoma compared to leiomyoma (20). Another study emphasized the prognostic value of VEGF in a large collection of soft tissue neoplasms but denied a correlation with MVD (21). Notably, the latter group employed the same antibody we used in our study. Another investigation found that in carcinosarcomas of the uterus, the MVD of the epithelial element was significantly higher than that of the mesenchymal element. Moreover, the epithelial component showed a higher VEGF expression than the mesenchymal element (22). In addition, no correlation with the MVD and the overall and relapse-free survival was found. Another study also failed to substantiate any relation between MVD and prognosis (23). Thus, it can be concluded that the parameter of MVD is not a predictor of malignant behavior or prognosis in soft tissue neoplasms.

Concerning VEGF receptor expression we observed a marked positivity for VEGFR-1 in the mononuclear infiltrate of myxoma and only a mildly positive infiltrate in the sarcoma group. The receptor was also present on the tumor cells themselves, as was VEGFR-2. The two receptors were less expressed in the sarcoma versus the myxoma group. These results emphasize that cardiac myxoma have a strong angiogenic potential driven by the release of VEGF through macrophages and the tumor cells as well as an autocrine activation of VEGF receptors on these cells, thereby perpetuating the angiogenic response within these tumors. This phenomenon has been described for different tumor types such as leukemia, lymphoma, melanoma or Kaposi sarcoma (24). Thus, the stronger expression of the receptors in myxoma compared to the sarcoma may explain the strong angiogenic response and consequently equal MVD compared to the sarcomas. The evaluation of PDGF-AA and its receptor PDGFR- $\alpha$ in the myxomas showed a unique and marked expression of the two molecules. To our knowledge, this is the first time that this PDGF receptor has been investigated in these tumors. Since there is a potent inhibitor available to block the signaling pathway of this growth factor these findings provide evidence that a recurrent or incurable myxoma Imatinib may be an additional therapeutic option for the patient. This drug has already been successfully tested in patients with Kaposi sarcoma or dermatofibrosarcoma protuberans (25). Notably, in the sarcoma group the receptor and its corresponding ligand were weakly expressed compared to the cardiac myxoma. However, it is important to emphasize that an immunohistochemical expression does not necessarily provide evidence for a functionally active growth factor receptor signal as was previously outlined. Thus, functional studies in the two entities are required to prove whether the signal disruption of the growth factor may lead to a significant anti-proliferative effect. Most cases in the myxoma and sarcoma group express PDGF-BB and its growth factor PDGFR- $\beta$. PDGFR- $\beta$ is also present on numerous pericytes. This is a common finding, since it is known that PDGFR- $\beta$ plays a pivotal role in the recruitment of pericytes to stabilize vascular endothelial cells and induce vascular maturation. Moreover, studies have provided strong evidence that blocking this process in experimental tumors may lead to a reduced tumor growth (8).

In summary, our study indicates that although myxoma and PAS are morphologically and pathophysiologically distinct entities with a different pathogenesis and clinical behavior they develop a comparable tumor microvascularisation. Hypoxia is a strong inducer of VEGF, leading to a higher vascular density than in other tumor areas. Receptor tyrosine kinases of the VEGF superfamily are present in the two tumor entities and may provide a basis for adjuvant therapy in the near future.

\section{Acknowledgements}

The authors wish to acknowledge the excellent technical support of Martina Waeber und Matthias Hornberg.

\section{References}

1. Amano J, Kono T, Wada Y, et al: Cardiac myxoma: its origin and tumor characteristics. Ann Thorac Cardiovasc Surg 9: 215-221, 2003.

2. Shapiro LM: Cardiac tumours: diagnosis and management. Heart 85: 218-222, 2001.

3. Pucci A, Gagliardotto P, Zanini C, et al: Histopathologic and clinical characterization of cardiac myxoma: review of 53 cases from a single institution. Am Heart J 140: 134-138, 2000. 
4. Burke AP and Virmani R: Sarcomas of the great vessels. A clinicopathologic study. Cancer 71: 1761-1773, 1993.

5. Folkman J: Role of angiogenesis in tumor growth and metastasis. Semin Oncol 29: 15-18, 2002.

6. Laakkonen P, Waltari M, Holopainen T, et al: Vascular endothelial growth factor receptor 3 is involved in tumor angiogenesis and growth. Cancer Res 67: 593-599, 2007.

7. Matsumoto $\mathrm{T}$ and Claesson-Welsh L: VEGF receptor signal transduction. Sci STKE 2001: RE21, 2001.

8. Bergers G, Song S, Meyer-Morse N, Bergsland E and Hanahan D: Benefits of targeting both pericytes and endothelial cells in the tumor vasculature with kinase inhibitors. J Clin Invest 111: 1287-1295, 2003.

9. Vermeulen PB, Gasparini G, Fox SB, et al: Second international consensus on the methodology and criteria of evaluation of angiogenesis quantification in solid human tumours. Eur J Cancer 38: 1564-1579, 2002.

10. Carmeliet P: VEGF as a key mediator of angiogenesis in cancer. Oncology 69: S4-S10, 2005

11. Kono T, Koide N, Hama Y, et al: Expression of vascular endothelial growth factor and angiogenesis in cardiac myxoma: a study of fifteen patients. J Thorac Cardiovasc Surg 119: 101-107, 2000

12. Sakamoto H, Sakamaki T, Kanda T, et al: Vascular endothelial growth factor is an autocrine growth factor for cardiac myxoma cells. Circ J 68: 488-493, 2004.

13. Salvesen HB and Akslen LA: Significance of tumour-associated macrophages, vascular endothelial growth factor and thrombospondin-1 expression for tumour angiogenesis and prognosis in endometrial carcinomas. Int J Cancer 84: 538-543, 1999.

14. Ohta M, Kitadai Y, Tanaka S, et al: Monocyte chemoattractant protein-1 expression correlates with macrophage infiltration and tumor vascularity in human gastric carcinomas. Int J Oncol 22: 773-778, 2003

15. Dankbar B, Padro T, Leo R, et al: Vascular endothelial growth factor and interleukin-6 in paracrine tumor-stromal cell interactions in multiple myeloma. Blood 95: 2630-2636, 2000.

16. Zhang T, Koide N, Wada Y, et al: Significance of monocyte chemotactic protein-1 and thymidine phosphorylase in angiogenesis of human cardiac myxoma. Circ J 67: 54-60, 2003.
17. Ramanathan M, Pinhal-Enfield G, Hao I and Leibovich SJ Synergistic up-regulation of vascular endothelial growth factor (VEGF) expression in macrophages by adenosine A2A receptor agonists and endotoxin involves transcriptional regulation via the hypoxia response element in the VEGF promoter. Mol Biol Cell 18: 14-23, 2007.

18. Hlatky L, Hahnfeldt P and Folkman J: Clinical application of antiangiogenic therapy: microvessel density, what it does and doesn't tell us. J Natl Cancer Inst 94: 883-893, 2002.

19. Chou CY, Huang SC, Tsai YC, Hsu KF and Huang KE: Uterine leiomyosarcoma has deregulated cell proliferation, but not increased microvessel density compared with uterine leiomyoma. Gynecol Oncol 65: 225-231, 1997.

20. Hata K, Hata T, Iida K and Miyazaki K: Expression of thymidine phosphorylase in uterine sarcoma and uterine leiomyoma: association with microvessel density and Doppler blood flow analysis. Ultrasound Obstet Gynecol 10: 54-58, 1997.

21. Yudoh K, Kanamori M, Ohmori K, et al: Concentration of vascular endothelial growth factor in the tumour tissue as a prognostic factor of soft tissue sarcomas. Br J Cancer 84: 1610-1615, 2001.

22. Emoto M, Iwasaki H, Ishiguro M, et al: Angiogenesis in carcinosarcomas of the uterus: differences in the microvessel density and expression of vascular endothelial growth factor between the epithelial and mesenchymal elements. Hum Pathol 30: 1232-1241, 1999.

23. West CC, Brown NJ, Mangham DC, Grimer RJ and Reed MW: Microvessel density does not predict outcome in high grade soft tissue sarcoma. Eur J Surg Oncol 31: 1198-1205, 2005.

24. Dias S, Hattori K, Heissig B, et al: Inhibition of both paracrine and autocrine VEGF/VEGFR-2 signaling pathways is essential to induce long-term remission of xenotransplanted human leukemias. Proc Natl Acad Sci USA 98: 10857-10862, 2001.

25. Sjoblom T, Shimizu A, O'Brien KP, et al: Growth inhibition of dermatofibrosarcoma protuberans tumors by the platelet-derived growth factor receptor antagonist STI571 through induction of apoptosis. Cancer Res 61: 5778-5783, 2001. 